\title{
Gender Differences in the Interest in Mathematics Schoolwork Across 50 Countries
}

\author{
Kimmo Eriksson ${ }^{1,2 *}$ \\ ${ }^{1}$ School of Education, Culture and Communication, Mälardalen University, Västerås, Sweden, ${ }^{2}$ Centre for Cultural Evolution, \\ Stockholm University, Stockholm, Sweden
}

Although much research has found girls to be less interested in mathematics than boys are, there are many countries in which the opposite holds. I hypothesize that variation in gender differences in interest are driven by a complex process in which national culture promoting high math achievement drives down interest in math schoolwork, with the effect being amplified among girls due to their higher conformity to peer influence. Predictions from this theory were tested in a study of data on more than 500,000 grade 8 students in 50 countries from the 2011 and 2015 waves of TIMSS. Consistent with predictions, national achievement levels were strongly negatively correlated with national levels of math schoolwork interest and this variation was larger among girls: girls in lowachievement, high-interest countries had especially high interest in math schoolwork, whereas girls in high-achievement, low-interest countries had especially low interest in math schoolwork. Gender differences in math schoolwork interest were also found to be related to gender differences in math achievement, emphasizing the importance of understanding them better.

Keywords: learning attitudes, gender differences, mathematics achievement, peer influence, female amplification

\section{INTRODUCTION}

Children and young adolescents are typically obliged to go to school and must take part in schoolwork even if they do not find it interesting. Nonetheless, it is preferable that students are interested in their schoolwork, both because they are likely to experience more satisfaction in school and because they are likely to achieve better (e.g., Artelt et al., 2003). Given these benefits of having high levels of interest, it is problematic that a large body of research has found that girls tend to have less interest in mathematics than boys do (Hyde et al., 1990; Lippa, 1998; Preckel et al., 2008; Su et al., 2009; Frenzel et al., 2010). However, this gender gap in mathematics interest does not seem to be universal. Recent research using cross-national data from the Trends in Mathematics and Science Survey (TIMSS) has uncovered that in many countries the gender gap in mathematics attitudes, including interest in schoolwork, is reversed (Ghasemi and Burley, 2019; Reilly et al., 2019). These findings suggest that the correct question to ask is not why girls are less interested in math than boys are, because often the opposite holds. From an egalitarian perspective, however, a gender gap in interest in a key subject in school may be regarded as equally problematic regardless of whether it favors boys or girls. Therefore, the present research aims to better understand why the level of interest in mathematics schoolwork may differ between the average boys and the average girls in a society develop, and why the difference may go either way depending on the society. 
The theoretical idea I propose is that gender differences in interest in schoolwork may be influenced by a societal factor-the achievement culture, which tends to drive interest in schoolwork down-in combination with a gender difference in conformity, with girls tending to conform more than boys. The outcome, I argue, would be a specific, complex pattern. In high-achievement cultures, it would be common for students to have a low level of interest in math schoolwork and, due to conformity, a low level of interest would be especially common among girls. In low-achievement cultures, by contrast, it would be common for students to have a high level of interest in math schoolwork and, again due to conformity, a high level of interest would be especially common among girls. Thus, high-achievement cultures would exhibit gender gaps in math schoolwork interest that favor boys, while gender gaps would be reversed in low-achievement cultures. Below I develop this novel hypothesis in greater detail, grounding its assumptions in previous literature.

\section{The Impact of High-Achievement Culture on Students' Math Schoolwork Interest}

The achievement culture of a society may be an important factor behind how interested students are in mathematics schoolwork. When comparing across countries, it is well-known that a high average level of student achievement in mathematics and science is related to a range of negative outcomes, including more negative attitudes to math and science (Artelt et al., 2003; Shen and Tam, 2008; Leung, 2014; Täht et al., 2014), worse student-teacher relations (Mikk et al., 2016), and worse academic self-concept among students (Shen and Tam, 2008; Van de gaer et al., 2012; Leung, 2014). For instance, on a global scale, math achievement is very low in Egypt and very high in Japan; nonetheless, a high positive affect toward mathematics was found to be very common among grade 8 students in Egypt but very uncommon in the same grade in Japan (see Table 21 in Leung, 2014). This phenomenon has been described as "paradoxical" (Shen and Tam, 2008). At the individual level, positive attitudes, good student-teacher relations, and a positive academic self-concept are generally regarded as conducive to learning (Artelt et al., 2003). Yet, a national culture that focuses on high achievement may bring about more negative attitudes, worse student-teacher relations, and a more negative academic self-concept. To explain this phenomenon, researchers have pointed to particularly high educational norms and standards in high-achievement countries (Van de gaer et al., 2012). Many students may struggle to fully meet these high standards, even though their achievement is high on a global scale, and this could account for the surprisingly low academic self-concept in highachievement countries (Van de gaer et al., 2012). The level of interest in schoolwork could similarly be driven down by high educational norms and standards. Students who are struggling to keep up with a progressively difficult curriculum in math could lose interest in doing the schoolwork to progress to even more advanced math, instead preferring to consolidate their knowledge. Consistent with this body of research, I therefore expect that a higher average level of math achievement in a society is linked to a lower average level of interest in math schoolwork.

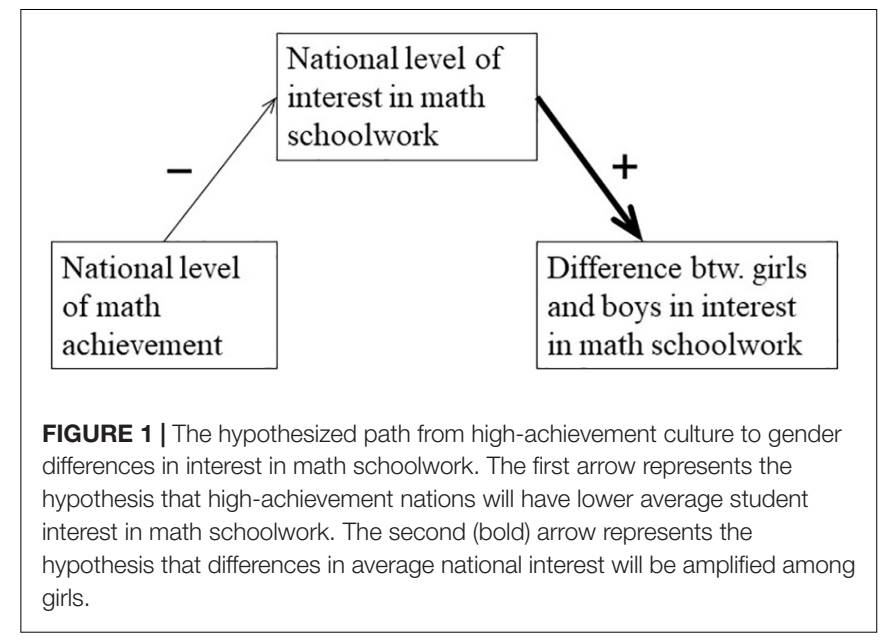

The first (fine) arrow in the diagram in Figure 1 illustrates this hypothesized negative relation.

\section{Peer Influence Among Girls and Boys}

It is well-known that students' motivation may be heavily influenced by their peers, both positively and negatively, and both intentionally and unintentionally (Kindermann, 2016; Wentzel and Muenks, 2016). Thus, if high-achievement culture makes some students lose interest in math schoolwork, it may negatively influence their peers' level of interest as well. Moreover, this effect of peer influence could differ between boys and girls. Studies of peer influence in school that have addressed the gender aspect have found that, compared to boys, girls' interest in schoolwork is more susceptible to peer influence (Berndt and Keefe, 1995; Riegle-Crumb et al., 2006). This effect has been attributed to girls' friendships being more supportive and discussion-oriented, compared to boys' friendships that tend to be more competitive and center more on specific activities (Riegle-Crumb et al., 2006; see also Beutel and Marini, 1995). Gender differences in the susceptibility to peer influence in school settings are consistent with findings from conformity research is general. Several metaanalyses have found conformity to be stronger among women than among men (Eagly and Carli, 1981; Bond and Smith, 1996). Although most of these studies have been carried out in Western countries, stronger conformity among women than among men was also observed in a recent study in Sudan when participants evaluated appropriate behavior (Efferson and Vogt, 2018). The gender effect on conformity has been explained in terms of women having less confidence and being more risk-averse than men (Cross et al., 2017; Brand et al., 2018).

Based on this previous literature I expect that within-society conformity with respect to interest in math schoolwork will be more accentuated among women than among men (through mechanisms such as gender differences in confidence, risk aversion, and friendships). However, the focus of the present research is on between-society variation, and greater conformity within societies is likely to lead to larger variation between societies. To understand this theoretical point, consider boys and girls in a high-achievement culture where some students 
lose their interest in math schoolwork due to high educational norms and standards. To some degree they will exert a negative influence on their peers' interest, and this peer influence is expected to be more pervasive among girls than among boys. Thus, the direct negative effect of high-achievement culture on students' interest is expected to be amplified by social dynamics, and this amplification is expected to be stronger among girls than among boys. The result would be greater variation across societies in the average interest levels of girls than in the average interest levels of boys.

This hypothesis is illustrated in the diagram in Figure 1, where the second (bold) arrow signifies the gender-specific amplification of national differences in interest in math schoolwork. Together, the two arrows describe a hypothetical indirect negative effect of a high national level of math achievement on gender differences in math schoolwork interest, mediated by the national level of math schoolwork interest.

\section{The Impact of Gender Differences in Math Schoolwork Interest on Math Achievement}

Gender differences in math schoolwork interest are important not least because they are likely to impact on the math achievement of boys and girls. At the individual level, interest in math schoolwork is thought to be conducive to learning (Artelt et al., 2003). In societies where girls tend to be less interested in math schoolwork than the boys are, it could contribute to a corresponding gender difference in math achievement. Why the gender gap in math achievement varies across societies has been the topic of extensive research for decades (e.g., Guiso et al., 2008; Else-Quest et al., 2010). A recent study, using data from all waves of the TIMSS and PISA assessments between 2000 and 2015 , found the societal level of gender egalitarian values to be the strongest and most robust predictor of gender differences in achievement in math, science, and reading, but there was still a large amount of unexplained variation (Eriksson et al., 2020). I hypothesize that some of this variation is accounted for by gender differences in interest in schoolwork. This would underscore the importance of the study's main aim of understanding why these gender differences vary across countries.

\section{Research Questions}

Above I have outlined a theory about antecedents and consequences of societal levels of math schoolwork interest among girls and boys. To fully test claims of causality would require experimental or, at least, longitudinal data, neither of which are available. Instead, I here make do with analyzing crosssectional data provided by TIMSS. The theory predicts certain statistical patterns to arise in such data and the aim of the empirical part of this study is to examine whether these patterns can indeed be observed. It is an important first test of the theory to see whether it correctly predicts several non-trivial features of a complex dataset, even though alternative causal accounts cannot be excluded.

RQ1. The hypothesis of high-achievement culture impacting on students' math schoolwork interest yields the first prediction to be examined: Is there a negative correlation between national levels of achievement and math schoolwork interest?

RQ2. The hypothesis of a difference between boys and girls in peer influence on math schoolwork interest yields a suite of testable predictions: (a) Is within-society variation in math schoolwork interest smaller among girls than among boys? (b) Is between-society variation in math schoolwork interest larger among girls than among boys? (c) Is there a positive correlation between national levels of math schoolwork interest and gender gaps in math schoolwork interest favoring girls? (d) Do national levels of math schoolwork interest mediate a negative correlation between national levels of achievement and gender gaps in math schoolwork interest favoring girls?

RQ3. The hypothesis that gender differences in math schoolwork interest has an independent impact on the gender gap in math achievement also yields a testable prediction: Does the gender gap in math schoolwork interest account for some of the variance in the gender gap in math achievement, over and beyond the variation already accounted for by gender egalitarian values?

\section{MATERIALS AND METHODS}

To answer the research questions, the current study analyzes TIMSS data. TIMSS is an excellent resource for comparative research as it uses large representative national samples of students from many countries. Details on the design are provided by the International Association for the Evaluation of Educational Achievement (Martin et al., 2016). In brief, TIMSS assesses math achievement of students in the eighth grade, in which most participants are about 14 years old. In addition to the achievement test, participating students also complete a background questionnaire. This questionnaire is not fixed across waves. In the 2011 and 2015 waves of TIMSS, the questionnaire included items on students' interest in what the teacher says and students' interest in what the teacher tells them to do. No such questions were included in previous waves of TIMSS, nor have they been included in other large-scale international student assessments like PISA. For this reason, this study will use data from the 2011 and 2015 waves of TIMSS.

Data from the 2011 and 2015 waves of TIMSS were downloaded from IEA ${ }^{1}$. Data were available for a total of 50 countries, out of which 35 countries had participated in both waves, 10 countries had participated only in the 2011 wave, and 5 countries only in the 2015 wave. See Table 1 for countries and samples sizes in each wave. All populated world continents were represented, including 24 countries in Asia from Israel and Saudi Arabia in the west to Korea and Japan in the east, 6 countries in Africa from Morocco and Egypt in the north to Botswana and South Africa in the south, 4 countries in the Americas from Canada to Chile, 14 countries in Europe from Sweden to Malta, as well as Australia and New Zealand.

The TIMSS datasets come with appropriate sampling weights, which were used when calculating the below measures. Missing data (less than $3 \%$ of data) were ignored. Preliminary analyses

\footnotetext{
${ }^{1}$ https://timssandpirls.bc.edu/
} 
TABLE 1 | TIMSS sample sizes and key measures.

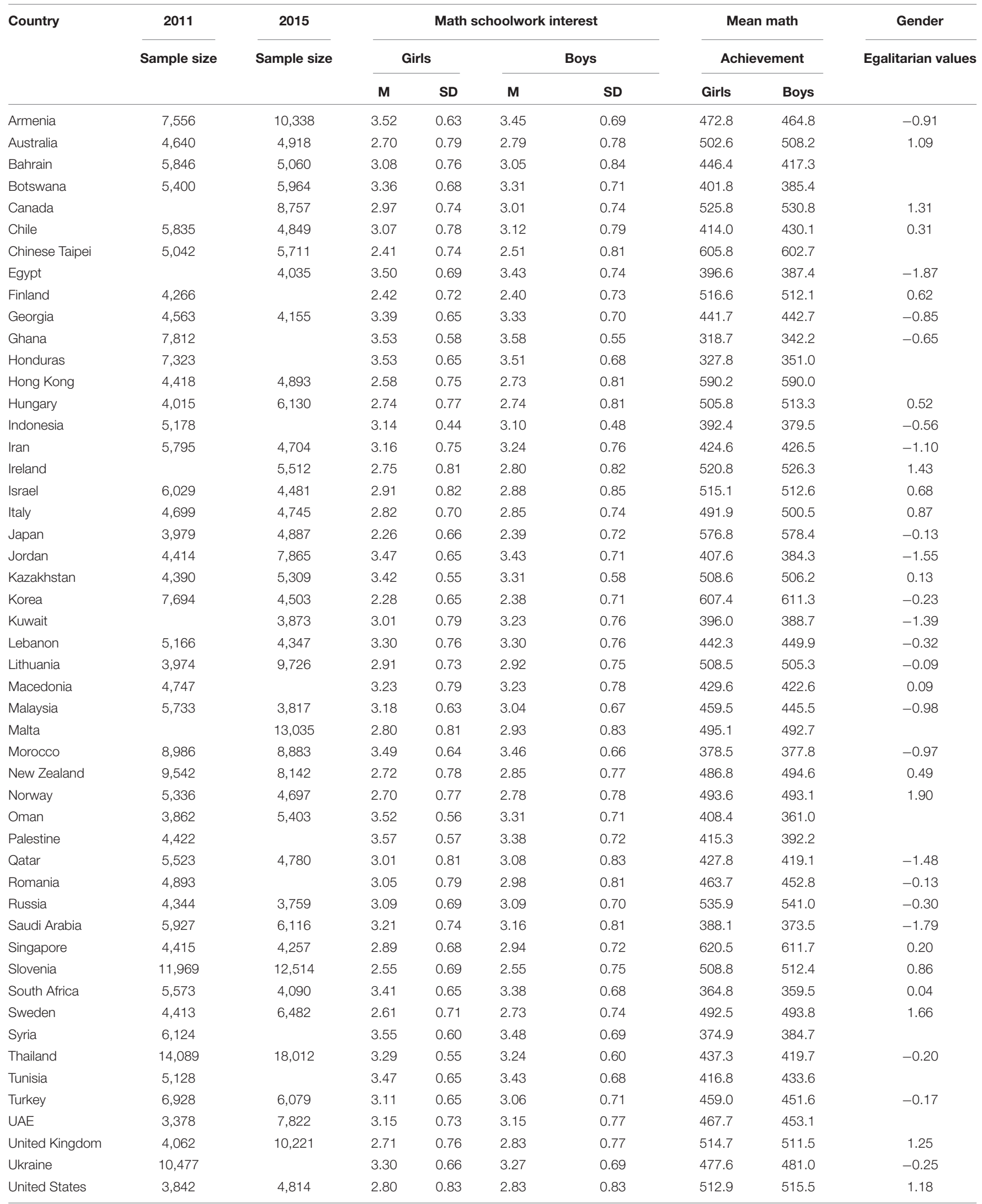

All measures are based on TIMSS data except the measure of gender egalitarian values, which is based on data from World Values Survey and GLOBE. 
revealed that country measures were highly consistent across the two waves. For the below analysis we therefore pooled the individual data from the two waves.

\section{Students' Interest in Math Schoolwork}

The student questionnaire in the 2011 and 2015 waves of TIMSS included three items bearing explicitly on interest in math schoolwork: "I am interested in what my teacher says," "My teacher gives me interesting things to do," and "I learn many interesting things in mathematics." For each item, students gave their response on a four-point scale: Disagree a lot (coded 1), Disagree a little (coded 2), Agree a little (coded 3), Agree a lot (coded 4). These three items were averaged to an internally consistent measure of students' interest in math schoolwork $(\alpha=0.78)$. Mean value and standard deviation, separately among girls and among boys, are reported per country in Table 1.

\section{Student Achievement in Mathematics}

TIMSS provides ready calculated national average scores for girls' and boys' math achievement, which were downloaded using the International Data Explorer of the National Center for Education Statistics $^{2}$ (see Table 1).

\section{Gender Egalitarian Values}

Following Eriksson et al. (2020), gender egalitarian values were measured using the Equality index from the World Values Survey (Welzel, 2013) and the Gender Egalitarian Cultural Values index from the GLOBE project (House et al., 2004). Both measures are based on survey responses to items on how society should be with respect to gender equality in education, leadership, and jobs. From Eriksson et al. (2020), measures of gender egalitarian values were obtained for 39 out of the 50 countries in the study: 26 countries had measures from both WVS and GLOBE, 11 countries only from WVS, and 2 countries only from GLOBE. On the set of 26 countries for which both measures were available, they were very strongly correlated, $r=0.85$, indicating that they indeed measure the same construct so that the measures can be combined. After transforming both WVS and GLOBE measures to z-scores (i.e., standardizing both measures to have the same mean value, zero, and the same standard deviation, one), I combined them into a single measure, using their average for any country where both measures were available.

\section{RESULTS}

Country levels of math schoolwork interest, general affect toward math, and math achievement, were calculated as the averages of the corresponding mean values for girls and boys. Gender differences for the same variables were similarly calculated as the mean value for girls minus the mean value for boys. Table 2 presents descriptive statistics of these national levels and gender differences.

${ }^{2}$ https://nces.ed.gov/timss/idetimss/
TABLE 2 | Descriptive statistics for country levels and gender differences of the measures in Table $\mathbf{1}$

\begin{tabular}{lcccc}
\hline Variable & $\boldsymbol{M}$ & SD & Min & Max \\
\hline Country level of math schoolwork interest & 3.06 & 0.34 & 2.33 & 3.55 \\
Country level of math achievement & 464.3 & 70.3 & 330 & 616 \\
Gender difference in math schoolwork interest & -0.01 & 0.09 & -0.22 & 0.20 \\
Gender difference in math achievement & 2.9 & 12.7 & -24 & 47
\end{tabular}

Based on $n=50$ countries. Country levels are calculated as the average of the mean values for girls and boys in Table 1, while gender differences are calculated as the girls' mean value minus the boys' mean value.

\section{RQ1: The Predicted Relation Between Country Levels of Achievement and Math Schoolwork Interest}

The first research question concerns the prediction of a negative correlation between country levels of achievement and math schoolwork interest. In line with the prediction, a very strong negative correlation was observed, $r(48)=-0.81,95 \% \mathrm{CI}$ $[-0.70,-0.89], p<0.001$. Here and throughout, I report bias corrected accelerated confidence intervals based on 1,000 bootstrap samples generated by SPSS v. 26.

\section{RQ2: Predictions Based on an Assumed Difference in Peer Influence on Girls' vs. Boys' Math Schoolwork Interest}

RQ2a: Is within-society variation in math schoolwork interest smaller among girls than among boys?

In Table 1, the standard deviation in math schoolwork interest was smaller among girls than among boys in 43 out of 50 countries, in line with the prediction. After transforming standard deviations to variances, the mean difference between girls $(M=0.497, S D=0.115)$ and boys $(M=0.545, S D=0.104)$ was $-0.048,95 \%$ CI $[-0.062,-0.034], t(49)=-6.93, p<0.001$, $d=0.98$, paired samples $t$-test.

\section{RQ2b: Is Between-Society Variation in Math Schoolwork Interest Larger Among Girls Than Among Boys?}

Figure 2 presents a scatterplot of girls' and boys' levels of interest in math schoolwork plotted against the average level. As indicated by the regression lines, girls in high-interest countries are even more interested than the boys are, while girls in low-interest countries are even less interested than the boys. Thus, between-society variation was larger for girls, in line with the prediction. To quantify the difference, the country variance of the math schoolwork interest level among girls was $\sigma^{2}=0.133$, which is $36 \%$ higher than the corresponding country variance among boys, $\sigma^{2}=0.098$. To estimate the statistical significance, we use the Morgan-Pitman test for difference in variance in paired data. This test assumes normally distributed data, and Kolmogorov-Smirnov tests indicated that the country level data on math schoolwork interest did not deviate from a normal distribution either for girls or boys, $p s>0.20$. The Morgan-Pitman test says that testing for a difference in variance in paired data is equivalent to testing 


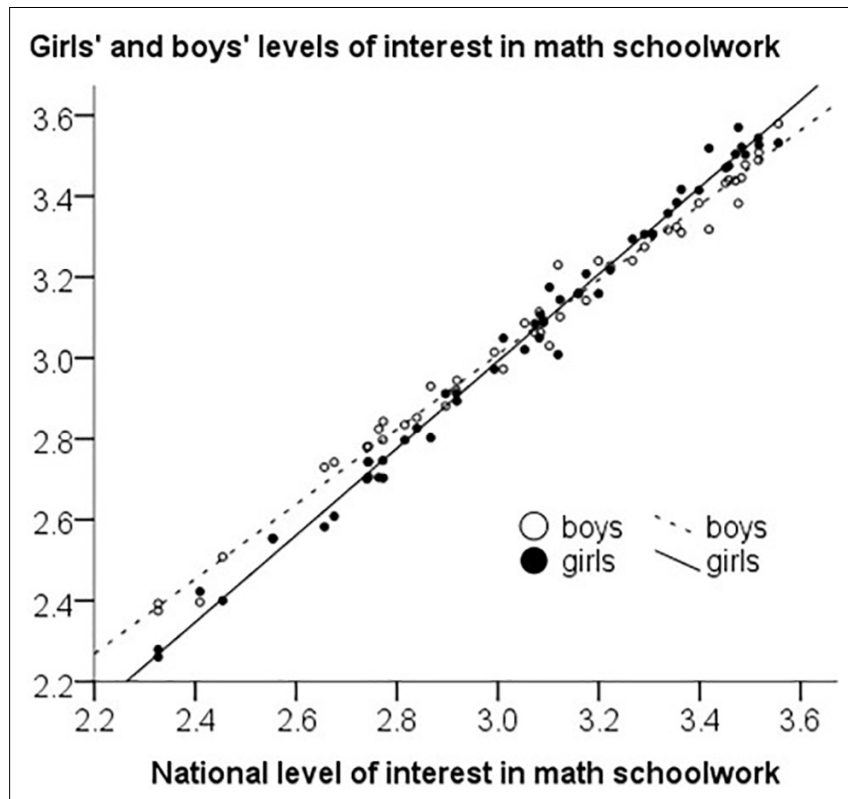

FIGURE 2 | Country variation in math schoolwork interest is amplified among girls. In high-interest countries, the interest levels of girls (black dots, solid line) tend to be higher than the interest levels of boys (circles, dashed regression line), while in low-interest countries girls tend to have lower interest levels than boys do.

for a correlation between the mean of the paired variables and the difference between the paired values (Wilcox, 2015). In our case, this means testing for a correlation between the total country level of math schoolwork interest and the gender difference in math schoolwork interest. In other words, research questions RQ2b and RQ2c are statistically equivalent. We conduct the test below.

RQ2c: Is There a Positive Correlation Between National Levels of Math Schoolwork Interest and Gender Gaps in Math Schoolwork Interest Favoring Girls?

In line with the prediction, there was a strong positive correlation between the total country level of math schoolwork interest and the gender difference in math schoolwork interest, $r(48)=0.60$, $95 \%$ CI $[0.39,0.78], p<0.001$. However, Kuwait was diagnosed as an outlier (standardized residual $>3$ ) (see Figure 3). If the outlier is excluded, the correlation is even higher, $r(47)=0.66$.

\section{RQ2d: Do National Levels of Math Schoolwork Interest Mediate a Negative Correlation Between National Levels of Achievement and Gender Gaps in Math Schoolwork Interest Favoring Girls?}

In line with the prediction, there was a negative correlation between national levels of math achievement and gender differences in math schoolwork interest, $r(48)=-0.45,95 \% \mathrm{CI}$ $[-0.64,-0.22], p=0.001$. To examine mediation, I employed the PROCESS macro, model 4, for SPSS (Hayes, 2017), after standardizing all variables to have unit standard deviation.
Results are reported in the mediation diagram in Figure 4, showing that the abovementioned correlation was fully mediated by the national interest in math schoolwork.

\section{RQ3. Does the Gender Gap in Math Schoolwork Interest Account for Variance in the Gender Gap in Math Achievement Unexplained by Gender Egalitarian Values?}

There was a positive correlation between gender differences in math schoolwork interest and gender differences in math achievement, $r(48)=0.46,95 \%$ CI $[0.15,0.68], p<0.001$. Results are virtually unchanged when the analysis is restricted to the subset of 39 countries for which measures of gender egalitarian are available, $r(37)=0.41,95 \%$ CI $[0.16,0.63], p=0.001$.

Consistent with prior research (Eriksson et al., 2020), gender egalitarian values were negatively correlated gender differences in achievement, $r(37)=-0.43,95 \%$ CI $[-0.66,-0.15], p<0.001$. On their own, gender egalitarian values accounted for $19 \%$ of the country variation in gender differences in math achievement. In line with the prediction, this proportion increased to $27 \%$ when the regression additionally included gender differences in math schoolwork interest, $\beta=0.31, p=0.049$.

\section{DISCUSSION}

The present paper studied the difference between girls and boys in their interest in math schoolwork and how it varies across countries. A theory was proposed according to which national culture promoting high math achievement drives down interest in math schoolwork, but more among girls than among boys due to conformity to peer influence being stronger among girls. Moreover, I argued that gender differences in math schoolwork interest are important because they will contribute to gender differences in math achievement. In the absence of experimental data, I tested the predictions this theory makes about statistical observations in cross-sectional data, provided by TIMSS. Results were consistent with predictions, as detailed below.

First, an extremely strong negative correlation between national levels of achievement and math schoolwork interest was observed. This finding, which is well in line with prior research on the relation between national achievement levels and attitudes to math and science (Shen and Tam, 2008; Täht et al., 2014), is consistent with the hypothesis that students' interest in schoolwork is negatively influenced by the high educational norms and standards in high-achievement cultures (Van de gaer et al., 2012). That high-achievement culture may be killing students' interest is arguably a serious problem. Comparisons between high-achieving countries indicate that the problem might be solvable, however. In a study of TIMSS data from 1999 to 2003, Shen and Tam (2008) pointed out that students in Singapore, an extremely high-achieving country, nonetheless had relatively positive attitudes toward math and science. Leung (2002) made the same observation. Singapore was a positive exception also in the current study, having the highest 


\section{Gender difference in interest in math schoolwork}

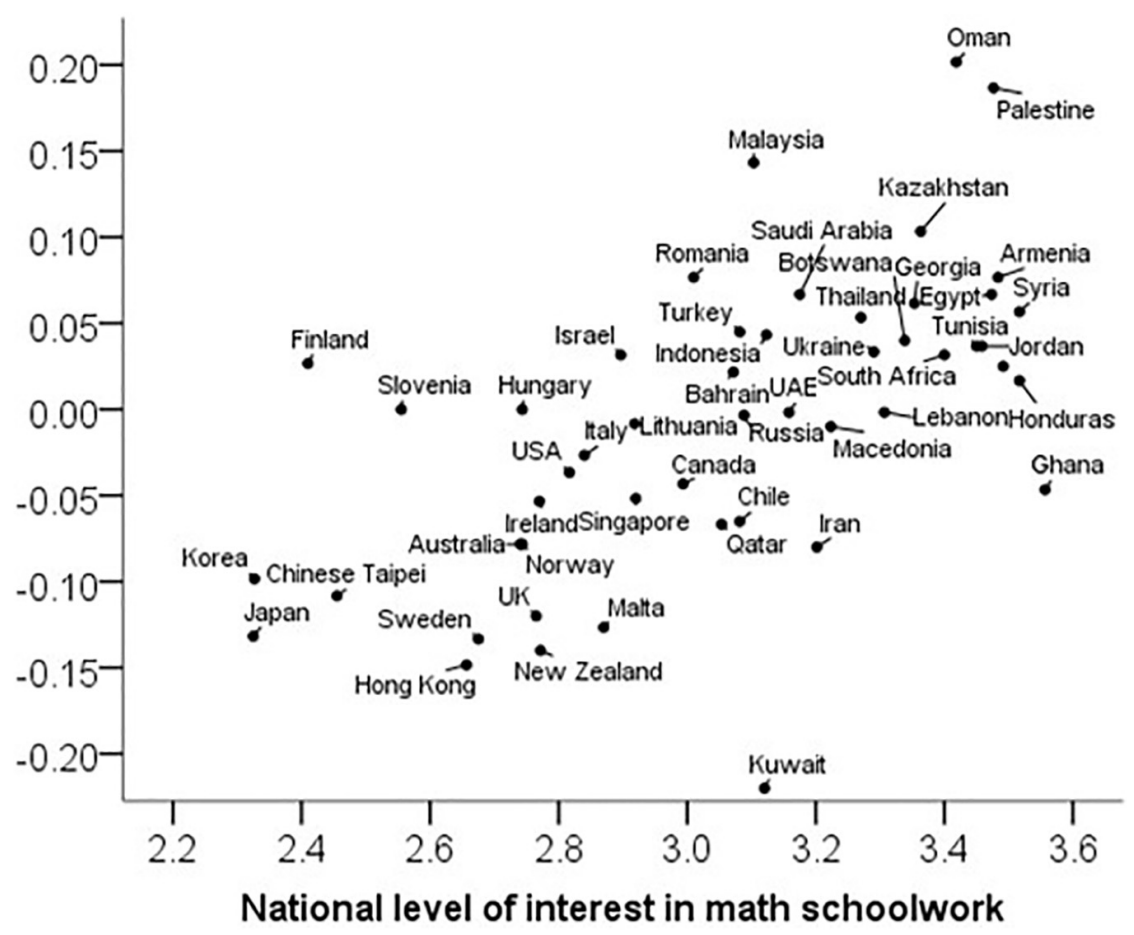

FIGURE 3 | Gender differences (favoring girls) in math schoolwork interest correlate with the national level of interest. The Pearson correlation is $r=0.60$ (or $r=0.66$ if the outlier Kuwait is excluded).

achievement level of all countries in the study, yet exhibiting a much higher national level of interest in math schoolwork than similarly high-achieving Korea and Japan did (Table 1). It would be valuable to understand whether there is some specific feature of Singapore's school system that mitigates the negative side effects of a high-achievement culture.

Second, several findings were consistent with the hypothesis that conformity to peer influence on math schoolwork interest is

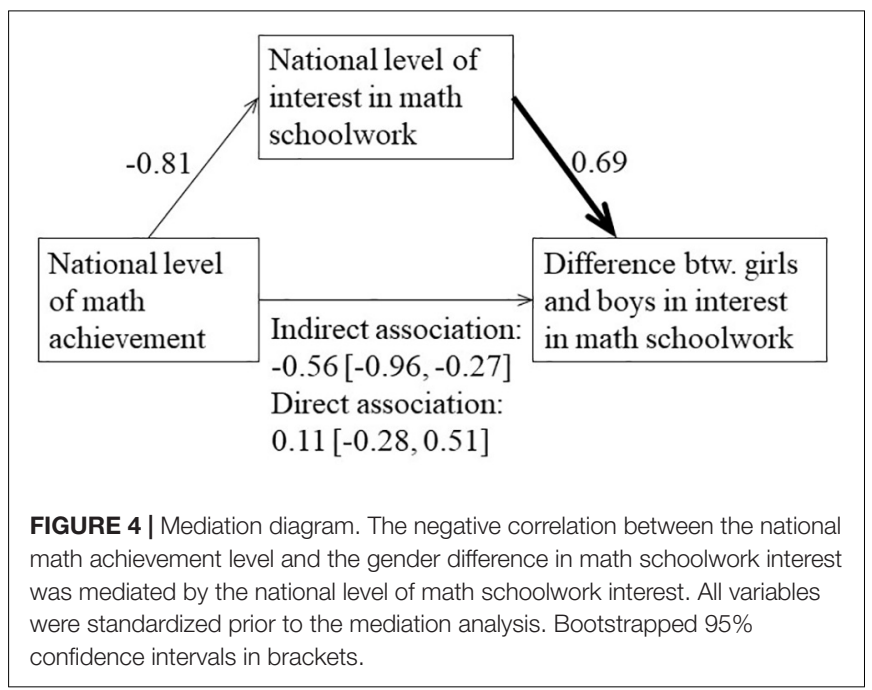

higher among girls than among boys. In almost all countries in the study, within-society variation in math schoolwork interest was smaller among girls than among boys, thus indicating greater female conformity. Because societies vary in their average level of interest in math schoolwork, the effect of peer pressure will vary too. Consistent with greater susceptibility to peer influence among girls, between-society variation in math schoolwork interest was larger among girls than among boys (Figure 2): In countries where the interest in math schoolwork was low, it tended to be especially low among girls. Similarly, in countries where the interest in math schoolwork was high, it tended to be especially high among girls. Thus, the country variation in students' interest in mathematics schoolwork was amplified among girls. The same phenomenon could be observed in terms of a positive correlation between national levels of math schoolwork interest and gender differences in math schoolwork interest favoring girls.

Taken together, my theory proposes a pathway in which highachievement culture drives down schoolwork interest, which through differential peer influence creates gender gaps in interest disfavoring girls. Consistent with this pathway, I found a negative correlation between national levels of achievement and gender gaps in math schoolwork interest favoring girls, and this correlation was mediated by the national level of math schoolwork interest.

Why is it important how girls' and boys' interest in math schoolwork vary across countries? For one thing, it is 
theoretically important to realize that the variation is substantial. In countries like Japan, Hong Kong, Sweden, and New Zealand, the interest level of the average girl was about 0.2 standard deviations lower than the interest of the average boy. These findings are consistent with research arguing for a fundamental gender difference in subject interest (e.g., Su et al., 2009). But this view appears to be contradicted by the finding of other societies, such as Oman, Malaysia, Palestine, and Kazakhstan, in which the gender gap is at least as wide but reversed.

Gender differences in math interest may also have real-life implications by influencing how girls achieve in mathematics relative to boys in the same country. Consistent with this hypothesis, I found that variation in the gender gap in math schoolwork interest accounts for part of the proportion of variance in the gender gap in math achievement that is not explained by variation in gender egalitarian values (Eriksson et al., 2020).

This study is an example of the benefits of using big data from large-scale assessments of student achievement to examine phenomena in educational psychology. A limitation, inherent in the reliance on cross-sectional data, is that directions of causality are not established. The findings are consistent with the proposed theory, but they could also have arisen from other mechanisms. It is helpful to consider what these alternative mechanisms could be. With respect to the strong negative correlation between national levels of achievement and schoolwork interest, it seems implausible that it would arise from low interest levels having a positive effect on achievement levels. Following Van de gaer et al. (2012), I proposed that high educational norms and standards have lower interest as an undesired side effect. However, there might be something else going on and perhaps more detailed insights into the abovementioned differences between Singapore and its East Asian neighbors could shed more light on this.

Similar reasoning applies to the amplification among girls of national variation in schoolwork interest. I proposed that this arises from differential conformity to peer influence, but it cannot be excluded that there is some alternative societal factor that causes girls' interest levels to be more extreme than the interest levels of boys. An interesting possibility for future research would be for large-scale assessments to provide some direct measures of peer influence (see also Eriksson et al., 2020).

The idea of conceiving of high-achievement culture as a factor behind gender differences has a precedent. In a study of PISA data, Mann and DiPrete (2016) found that the national achievement level correlated with gender differences in academic self-concept and STEM aspirations. However, they did not examine the female amplification account, that is, whether these

\section{REFERENCES}

Artelt, C., Baumert, J., Julius-McElvany, N., and Peschar, J. (2003). Learners for life: Student approaches to learning. Results from PISA 2000. Paris: Organisation for Economic Co-operation and Development.

Berndt, T. J., and Keefe, K. (1995). Friends' influence on adolescents' adjustment to school. Child Dev. 66, 1312-1329. doi: 10.1111/j.1467-8624.1995.tb0 0937.x effects were mediated by national levels of academic self-concept and STEM aspirations. Future research should examine the scope of female amplification as a mechanism behind gender differences in various beliefs and attitudes.

To conclude, the present study has contributed to scientific understanding of gender differences in interest in mathematics schoolwork by, first, proposing a theory of why such gender differences would arise and vary across countries, and second, testing several theoretical predictions in a large cross-national dataset. Results were consistent with both key components of the theory: high-achievement culture may be detrimental to interest in schoolwork and this effect may be amplified among girls due to their higher conformity to peer influence. These positive findings motivate further study of the validity and scope of the proposed mechanisms.

\section{DATA AVAILABILITY STATEMENT}

Publicly available datasets were analyzed in this study. This data can be found here: https://osf.io/dwk8h/.

\section{ETHICS STATEMENT}

Ethical review and approval was not required for the study on human participants in accordance with the local legislation and institutional requirements. Written informed consent from the participants' legal guardian/next of kin was not required to participate in this study in accordance with the national legislation and the institutional requirements.

\section{AUTHOR CONTRIBUTIONS}

KE performed the statistical analysis and wrote the manuscript.

\section{FUNDING}

This research was supported by the Swedish Research Council (Grant No. 2014-2008) and the Knut and Alice Wallenberg Foundation (Grant No. 2015.0005).

\section{ACKNOWLEDGMENTS}

Ksenia Startseva-Lora provided helpful preliminary analyses. 
Cross, C. P., Brown, G. R., Morgan, T. J., and Laland, K. N. (2017). Sex differences in confidence influence patterns of conformity. Br. J. Psychol. 108, 655-667. doi: 10.1111/bjop.12232

Eagly, A. H., and Carli, L. L. (1981). Sex of researchers and sex-typed communications as determinants of sex differences in influenceability: a metaanalysis of social influence studies. Psychol. Bull. 90, 1-20. doi: 10.1037/00332909.90.1.1

Efferson, C., and Vogt, S. (2018). Behavioural homogenization with spillovers in a normative domain. Proc. R. Soc. B Biol. Sci. 285:20180492. doi: 10.1098/rspb. 2018.0492

Else-Quest, N. M., Hyde, J. S., and Linn, M. C. (2010). Cross-national patterns of gender differences in mathematics: a meta-analysis. Psychol. Bull. 136, 103-127. doi: 10.1037/a0018053

Eriksson, K., Björnstjerna, M., and Vartanova, I. (2020). The relation between gender egalitarian values and gender differences in academic achievement. Front. Psychol. 11:236. doi: 10.3389/fpsyg.2020.00236

Frenzel, A. C., Goetz, T., Pekrun, R., and Watt, H. M. (2010). Development of mathematics interest in adolescence: influences of gender, family, and school context. J. Res. Adolesc. 20, 507-537. doi: 10.1111/j.1532-7795.2010.00645.x

Ghasemi, E., and Burley, H. (2019). Gender, affect, and math: a cross-national meta-analysis of Trends in International Mathematics and Science Study 2015 outcomes. Large Scale Assess. Educ. 7:10. doi: 10.1186/s40536-019-0078-1

Guiso, L., Monte, F., Sapienza, P., and Zingales, L. (2008). Culture, gender, and math. Science 320, 1164-1165. doi: 10.1126/science.1154094

Hayes, A. F. (2017). Introduction to Mediation, Moderation, and Conditional Process Analysis: A Regression-Based Approach. New York, NY: Guilford publications.

House, R. J., Hanges, P. J., Javidan, M., Dorfman, P. W., and Gupta, V. (2004). Culture, Leadership, and Organizations: The GLOBE Study of 62 Societies. Thousand Oaks: SAGE Publications.

Hyde, J. S., Fennema, E., Ryan, M., Frost, L. A., and Hopp, C. (1990). Gender comparisons of mathematics attitudes and affect: a meta-analysis. Psychol. Women Q. 14, 299-324. doi: 10.1111/j.1471-6402.1990.tb00022.x

Kindermann, T. A. (2016). "Peer group influences on students' academic motivation," in Handbook of Social Influences in School Contexts: SocialEmotional, Motivation, and Cognitive Outcomes, eds G. B. Ramani, and K. R. Wentzel (Abingdon: Routledge), 31-47.

Leung, F. K. (2002). Behind the high achievement of East Asian students. Educ. Res. Eval. 8, 87-108. doi: 10.1076/edre.8.1.87.6920

Leung, F. K. (2014). What can and should we learn from international studies of mathematics achievement? Math. Educ. Res. J. 26, 579-605. doi: 10.1007/ s13394-013-0109-0

Lippa, R. A. (1998). Gender-related individual differences and the structure of vocational interests: the importance of the people-things dimension. J. Pers. Soc. Psychol. 74, 996-1009. doi: 10.1037/0022-3514.74.4.996

Mann, A., and DiPrete, T. A. (2016). The consequences of the national math and science performance environment for gender differences in STEM aspiration. Sociol. Sci. 3, 568-603. doi: 10.15195/v3.a25
Martin, M. O., Mullis, I. V. S., and Hooper, M. (eds) (2016). Methods and Procedures in TIMSS 2015. Chestnut Hill, MA: TIMSS \& PIRLS International Study Center, Lynch School of Education, Boston College.

Mikk, J., Krips, H., Säälik, Ü, and Kalk, K. (2016). Relationships between student perception of teacher-student relations and PISA results in mathematics and science. Int. J. Sci. Math. Educ. 14, 1437-1454. doi: 10.1007/s10763-015-9669-7

Preckel, F., Goetz, T., Pekrun, R., and Kleine, M. (2008). Gender differences in gifted and average-ability students: comparing girls' and boys' achievement, self-concept, interest, and motivation in mathematics. Gifted Child Q. 52, 146-159. doi: 10.1177/0016986208315834

Reilly, D., Neumann, D. L., and Andrews, G. (2019). Investigating gender differences in mathematics and science: results from the 2011 trends in mathematics and science survey. Res. Sci. Educ. 49, 25-50. doi: 10.1007/s11165017-9630-6

Riegle-Crumb, C., Farkas, G., and Muller, C. (2006). The role of gender and friendship in advanced course taking. Sociol. Educ. 79, 206-228. doi: 10.1177/ 003804070607900302

Shen, C., and Tam, H. P. (2008). The paradoxical relationship between student achievement and self-perception: a cross-national analysis based on three waves of TIMSS data. Educ. Res. Eval. 14, 87-100. doi: 10.1080/13803610801896653

$\mathrm{Su}, \mathrm{R}$., Rounds, J., and Armstrong, P. I. (2009). Men and things, women and people: a meta-analysis of sex differences in interests. Psychol. Bull. 135, 859-884. doi: $10.1037 / \mathrm{a} 0017364$

Täht, K., Must, O., Peets, K., and Kattel, R. (2014). Learning motivation from a cross-cultural perspective: a moving target? Educ. Res. Eval. 20, 255-274. doi: 10.1080/13803611.2014.929009

Van de gaer, E., Grisay, A., Schulz, W., and Gebhardt, E. (2012). The reference group effect: an explanation of the paradoxical relationship between academic achievement and self-confidence across countries. J. Cross Cult. Psychol. 43, 1205-1228. doi: 10.1177/0022022111428083

Welzel, C. (2013). Freedom Rising. Cambridge, MA: Cambridge University Press. Wentzel, K. R., and Muenks, K. (2016). "Peer influence on students' motivation, academic achievement, and social behavior," in Handbook of Social Influences in School Contexts: Social-Emotional, Motivation, and Cognitive Outcomes, eds G. B. Ramani, and K. R. Wentzel (Abingdon: Routledge), 13-30.

Wilcox, R. (2015). Comparing the variances of two dependent variables. J. Stat. Distrib. Applicat. 2:7. doi: 10.1186/s40488-015-0030-z

Conflict of Interest: The author declares that the research was conducted in the absence of any commercial or financial relationships that could be construed as a potential conflict of interest.

Copyright (c) 2020 Eriksson. This is an open-access article distributed under the terms of the Creative Commons Attribution License (CC BY). The use, distribution or reproduction in other forums is permitted, provided the original author(s) and the copyright owner(s) are credited and that the original publication in this journal is cited, in accordance with accepted academic practice. No use, distribution or reproduction is permitted which does not comply with these terms. 